\title{
SBC2012-XXXXXX
}

\section{ESTIMATED MUSCLE LOADS DURING SQUAT EXERCISE IN MICROGRAVITY CONDITIONS}

\author{
Christopher D. Fregly (1), Brandon T. Kim (1), Zhao Li (1), \\ John K. De Witt (2), and Benjamin J. Fregly (3)
}

\author{
(1) Lyceum Magnet Program, Abraham Lincoln \\ Middle School, Gainesville, FL 32641 USA
}

\author{
(2) Wyle Science, Technology and Engineering \\ Group, Houston, TX 77058 USA
}

(3) Dept. of Mechanical \& Aerospace Engineering, University of Florida, Gainesville, FL 32611 USA

\section{INTRODUCTION}

Loss of muscle mass in microgravity is one of the primary factors limiting long-term space flight [1]. NASA researchers have developed a number of exercise devices to address this problem. The most recent is the Advanced Resistive Exercise Device (ARED) [2], which is currently used by astronauts on the International Space Station (ISS) to emulate typical free-weight exercises in microgravity. ARED exercise on the ISS is intended to reproduce Earth-level muscle loads, but the actual muscle loads produced remain unknown as they cannot currently be measured directly.

In this study we estimated muscle loads experienced during squat exercise on ARED in microgravity conditions representative of Mars, the moon, and the ISS. The estimates were generated using a subjectspecific musculoskeletal computer model and ARED exercise data collected on Earth. The results provide insight into the capabilities and limitations of the ARED machine.

\section{METHODS}

Movement data were collected from a single male subject during ARED squat exercise performed at NASA Johnson Space Center. Institutional review board approval and informed consent were obtained. Data included surface marker positions measured by a 12camera motion capture system (SMART-D, BTS Bioengineering S.p.A., Milan, Italy) and ground reaction forces and moments measured by two force plates (Model 9261, Kistler Instruments AG, Winterthur, Switzerland). Experiment conditions included a normal parallel squat motion and four motion variations (increased hip and knee range of motion, decreased hip and knee range of motion, increased stance width, and faster squat).

Musculoskeletal modeling and simulation work was performed using OpenSim [3]. A nominal published walking model [4] was obtained from the Simtk.org website as a starting point for the analyses. The three-dimensional model initially possessed 37 degrees of freedom. All upper-body joints and both toe joints were locked in positions consistent with the squat motions, leaving 21 degrees of freedom. The joint structure of the nominal model was changed by replacing the ground-to-pelvis joint with a ground-to-shoulders joint. This change allowed OpenSim to calculate inverse dynamics loads at the shoulders rather than the pelvis using a bottom-up approach.

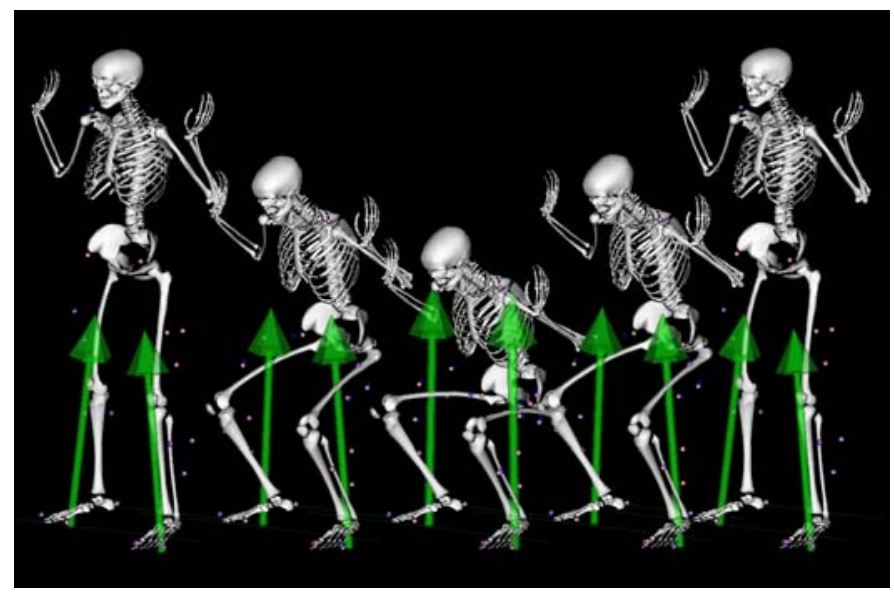

Fig 1: Subject motion and ground reaction forces for the normal ARED squat trial as produced by model scaling, inverse kinematics, and inverse dynamics in Opensim.

Analysis of the experimental ARED data involved a three-step process. First, model scaling using static trial marker data was performed to convert the nominal model into a subject-specific model. 
Second, inverse kinematics was performed using the subject-specific model to convert marker motion data into corresponding joint motions. Third, inverse dynamics was performed using the subject-specific model, joint motions from inverse kinematics, and ground reaction force measurements to calculate muscle moments at the ankle, knee, hip, and back joints and applied force on the shoulders (Fig. 1).

Inverse dynamic analyses were performed for the different gravity and squat variations. Gravity variations were analyzed using the joint motions and ground reactions from the normal squat trial. Reduced gravity was imposed by setting the gravity term in the model to $0.38 \mathrm{~g}$ for Mars, $0.17 \mathrm{~g}$ for the moon, and $0 \mathrm{~g}$ for the ISS. The four squat variations were analyzed using the joint motions and ground reactions from the corresponding experiment trials. The output of each inverse dynamic analysis was the time history of shoulder force and of each muscle moment. For each of these loads, the largest value in the squat cycle was selected for analysis. Muscle moments from the right and left legs were similar and were averaged during analysis.

\section{RESULTS}

For the different gravitational conditions, the model predicted similar ankle and knee loads but larger hip, back, and shoulder loads on Mars, the moon, and the ISS than on Earth (Table 1). The increase in shoulder force for each microgravity condition was almost exactly proportional to the decrease in bodyweight (BW) for that condition. Increases in maximum ankle, knee, hip, back, and shoulder loads were much larger when gravity was varied than when the squat exercise was varied. For the normal squat motion performed on Earth, maximum muscle moments were $49 \mathrm{Nm}$ for the ankle, $158 \mathrm{Nm}$ for the knee, 193 $\mathrm{Nm}$ for the hip, and $362 \mathrm{Nm}$ for the back, with the model-predicted shoulder force (1.31 BW) being consistent with the force applied by the ARED machine (1.27 BW).

Table 1: Absolute and percent increases in peak joint loads relative to Earth (1G) for simulated ARED squat exercise in various microgravity conditions.

\begin{tabular}{lcccc}
\hline & & \multicolumn{4}{c}{ Microgravity Condition } \\
Joint Load & & $\begin{array}{c}\text { Mars } \\
\text { Moon }\end{array}$ & $\begin{array}{c}\text { ISS } \\
\text { (0.38G) }\end{array}$ & $\begin{array}{c}\mathbf{( 0 . 1 7 G )} \\
\text { (0G) }\end{array}$ \\
\hline \multirow{2}{*}{ Ankle Moment } & $(\mathrm{Nm})$ & 0 & 1 & 1 \\
& $(\%)$ & $0 \%$ & $2 \%$ & $2 \%$ \\
\hline \multirow{2}{*}{ Knee Moment } & $(\mathrm{Nm})$ & 3 & 4 & 5 \\
& $(\%)$ & $2 \%$ & $3 \%$ & $3 \%$ \\
\hline \multirow{2}{*}{ Hip Moment } & $(\mathrm{Nm})$ & 33 & 43 & 48 \\
& $(\%)$ & $17 \%$ & $22 \%$ & $25 \%$ \\
\hline \multirow{2}{*}{ Back Moment } & $(\mathrm{Nm})$ & 47 & 60 & 71 \\
& $(\%)$ & $13 \%$ & $17 \%$ & $20 \%$ \\
\hline \multirow{2}{*}{ Shoulder Force } & $(\mathrm{BW})$ & 0.61 & 0.82 & 1.00 \\
& $(\%)$ & $46 \%$ & $62 \%$ & $76 \%$ \\
\hline
\end{tabular}

\section{DISCUSSION}

The model's predictions suggest that no single ARED shoulder force on the ISS will produce Earth-equivalent ankle, knee, hip, and back loads simultaneously. Increased shoulder force on the ISS was predicted to produce higher joint loads than on Earth, with the increases growing from the ankle (smallest increase) toward the back (largest increase). The shoulder force decrease needed to achieve Earth-equivalent back and hip loads would result in knee and ankle loads below Earth-equivalent levels. Thus, the optimal approach may be to choose a squat load that targets a specific joint of interest. A design modification that could be considered is to add bungee cords to the pelvis to pull it toward the ARED foot plate, similar to the bungee cords used with the ISS treadmill. Such a change could help distribute forces better throughout the body, reducing the shoulder force currently used on the ISS while achieving closer to Earth-equivalent muscle moments at all joints simultaneously.
Our results can be understood by considering the changes in joint loads relative to standing on Earth that would occur if a $1 \mathrm{BW}$ equivalent force was added to the shoulders while standing in $0 \mathrm{~g}$. The shoulders would experience a force increase of $1 \mathrm{BW}$, since no force is applied to the shoulders during standing on Earth. At the other extreme, the ankles would experience a force increase of only 0.03 BW, since the ankles support $97 \%$ of BW during standing on Earth. Thus, adding a $1 \mathrm{BW}$ force to the shoulders in $0 \mathrm{~g}$ will change Earthlevel joint loads by the amount of BW below the joint of interest.

Relative to the normal squat exercise, the technique variations generally had little effect on maximum muscle moments (data not shown). In contrast, every reduced-gravity case produced increased joint loads. Thus, the squat motion variation benefits when performed on the ISS are most probably related to exposing the musculoskeletal system to loading while in different kinematic positions.

Our modeling approach had two major limitations. First, we assumed that joint motions and ground reaction forces and moments measured on Earth would be the same in microgravity conditions. Though no quantitative data exist to evaluate these assumptions, a NASA video available on YouTube ("How Space Exploration Affects Muscles") suggests that astronauts perform ARED squat exercise differently on the ISS than they do on Earth. Second, we assumed that back motion of the human subject could be represented using a single ball-and-socket joint. In reality, the subject's back probably flexed at multiple locations, which may have affected the back moment calculations.

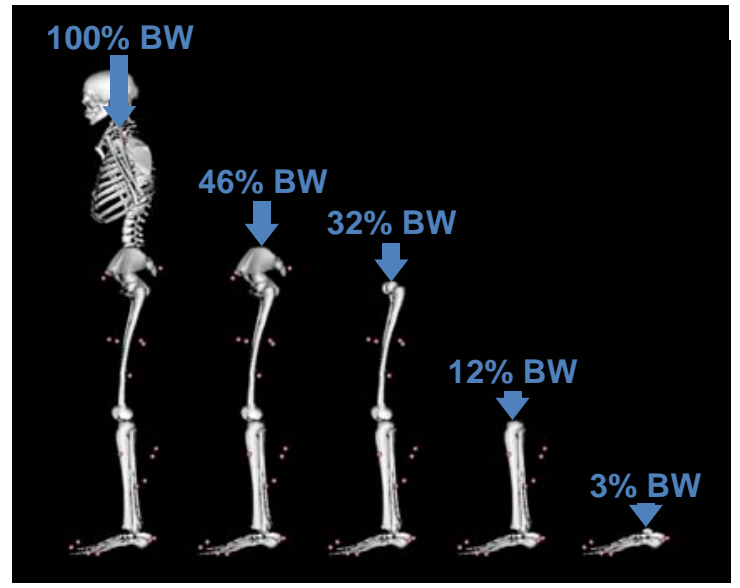

Fig. 2: Change in bodyweight (BW) relative to Earth at each joint level caused by adding a 100\% BW equivalent force to the shoulders on the ISS.

\section{ACKNOWLEDGMENTS}

The authors thank NASA Johnson Space Center for providing the ARED experiment movement data used in this study.

\section{REFERENCES}

1. Genc, K.O., et al., 2010, "Foot Forces during Exercise on the International Space Station,” J Biomech, 43, pp. 3020-3027.

2. Loehr, J.A., et al., 2010, "Musculoskeletal Adaptations to Training with the Advanced Resistive Exercise Device,” Med Sci Sports Exer, 43, 146-156.

3. Delp, S.L., et al., 2007, “OpenSim: Open-source Software to Create and Analyze Dynamic Simulations of Movement,” IEEE Trans Biomed Eng, 54, pp. 1940-1950.

4. Hamner, S.R., et al., 2010, "Muscle Contributions to Propulsion and Support during Running,” J Biomech, 43, pp. 2709-2716. 